\title{
Initialization and read-out of intrinsic spin defects in a van der Waals crystal at room temperature
}

Andreas Gottscholl1, Mehran Kianinia ${ }^{2}$, Victor Soltamov ${ }^{1}$, Carlo Bradac ${ }^{2}$, Christian Kasper ${ }^{1}$, Klaus Krambrock ${ }^{3}$, Andreas Sperlich ${ }^{1}$, Milos Toth², Igor Aharonovich ${ }^{2, *}$, Vladimir Dyakonov ${ }^{1, *}$

${ }^{1}$ Experimental Physics $\mathrm{VI}$ and Würzburg-Dresden Cluster of Excellence ct.qmat, Julius Maximilian University of Würzburg, 97074 Würzburg, Germany

2 School of Mathematics and Physical Sciences, University of Technology Sydney, Ultimo, NSW 2007, Australia

${ }^{3}$ Departamento de Física, Universidade Federal de Minas Gerais (UFMG), Belo Horizonte, MG, Brazil

Correspondence to igor.aharonovich@uts.edu.au or vladimir.dyakonov@uni-wuerzburg.de

\begin{abstract}
Optically addressable spins in wide-bandgap semiconductors have become one of the most prominent platforms for exploring fundamental quantum phenomena. While several candidates in 3D crystals including diamond and silicon carbide have been extensively studied, the identification of spindependent processes in atomically-thin $2 D$ materials has remained elusive. Although optically accessible spin states in $h B N$ are theoretically predicted, they have not yet been observed experimentally. Here, employing rigorous electron paramagnetic resonance techniques and photoluminescence spectroscopy, we identify fluorescence lines in hexagonal boron nitride associated with a particular defect - the negatively charged boron vacancy $\left(V_{B}^{-}\right)$- and determine the parameters of its spin Hamiltonian. We show that the defect has a triplet $(S=1)$ ground state with a zero-field splitting of $\approx 3.5 \mathrm{GHz}$ and establish that the centre exhibits optically detected magnetic resonance (ODMR) at room temperature. We also demonstrate the spin polarization of this centre under optical pumping, which leads to optically induced population inversion of the spin ground state - a prerequisite for coherent spin-manipulation schemes. Our results constitute a leap forward in establishing twodimensional hBN as a prime platform for scalable quantum technologies, with extended potential for spin-based quantum information and sensing applications.
\end{abstract}

The emergence of 2D materials and van der Waals ( $\mathrm{vdW}$ ) crystals has enabled the observation and realisation of unique optoelectronic and nanophotonic effects such as Moiré excitons and quantum spin Hall effect at elevated temperatures, to name a few ${ }^{1,2}$. Amidst the large variety of studied vdW crystals, hexagonal Boron Nitride (hBN) offers a combination of unique physical, chemical and optical properties $^{3}$. Most relevant to this work, is the ability of hBN to host atomic impurities (or point defects), that give rise to quantized optical transitions, well below its bandgap ${ }^{4,5}$. hBN colour centres are ultrabright with narrow and tuneable linewidth ${ }^{6-8}$, and photostability up to $800 \mathrm{~K}^{9}$. Whilst the nature of many of the defects is still uncertain, ${ }^{10-15}$ they are being extensively studied as promising candidates for quantum photonic applications requiring on-demand, ultrabright single-photon emission.

A step forward, which will significantly extend the functionality of hBN emitters for quantum applications, is to interface their optical properties with spin transitions, and realise spin-polarization and optical spinreadout schemes ${ }^{16,17}$. The concept of spin-photon interface has been extensively studied in quantum $\operatorname{dots}^{18}$ and the nitrogen-vacancy (NV) centre in diamond ${ }^{19}$. The latter has been harnessed to realise basic two-node quantum networks ${ }^{19}$ and a plethora of advanced quantum sensing schemes ${ }^{20-22}$. The basic principle is that the defect's high-spin ground or excited state can be polarised, manipulated and read out optically owing to the spin-dependent excitation, decay and intersystem crossing pathways available to the system during the optical excitation-recombination cycle ${ }^{23}$.

Yet, extending the optical control of single-spin states beyond defects in 3D crystals, to 2D systems, has remained elusive. If achieved, it will open up a stretch of novel possibilities both fundamental and technological. The two-dimensional nature of these materials inherently allows for seamless integration with heterogeneous, opto-electronic devices where the hosted solid-state qubits can be readily 
interfaced with cavities, resonators and nanophotonic components from foreign materials. Further, it naturally grants nanoscale proximity of the spin probe to target samples for high-resolution quantum sensing realizations. Reliable and deterministic transfer of $\mathrm{hBN}$ layers on stacks of other $2 \mathrm{D}$ materials is well-established and is part of one of the currently most relevant endeavours of condensed matter physics - engineering heterostructures made by purposefully-chosen sequences of atomically-thin 2Dmaterials ${ }^{24}$.

Here we report on the optical initialisation and readout of an ensemble of spins in hBN. We perform rigorous electron paramagnetic resonance (EPR) spectroscopy and optically detected magnetic resonance (ODMR) measurements to establish that the defect has a triplet ground state with zero field splitting (ZFS) of $\approx 3.5 \mathrm{GHz}$ and almost isotropic Landé factor $g=2.000$. From the analysis of the angular dependence and nitrogen hyperfine structure, we confirm the intrinsic nature of the defect and assign it to the boron lattice position, most likely being the negatively-charged boron vacancy $\left(V_{B}^{-}\right)$. The alternative substitutional carbon on a boron cite $\left(C_{B}\right)$ or nitrogen vacancy $\left(V_{N}\right)$ structures were also considered, but discarded upon analysis of the experimental data (see discussion below).

Figure $1 \mathrm{a}$ is a schematic illustration of the proposed defect. The defect is a negatively charged boron vacancy $\left(V_{B}^{-}\right)$centre consisting of a missing boron atom surrounded by three equivalent nitrogen atoms in the hBN lattice. The defect has $D_{3 h}$ point-group symmetry and exhibits strong room temperature photoluminescence (PL) emission at $\lambda_{\max } \approx 850 \mathrm{~nm}$ under $\lambda_{\text {exc }}=532 \mathrm{~nm}$ laser excitation with an excited state lifetime of $\tau_{\text {exc }}=1.2 \mathrm{~ns}$ (Figure $1 \mathrm{~b}$ ). Note, these defects are different than the quantum emitters around $2 \mathrm{eV}$ that were previously investigated ${ }^{5-8,12}$.

a

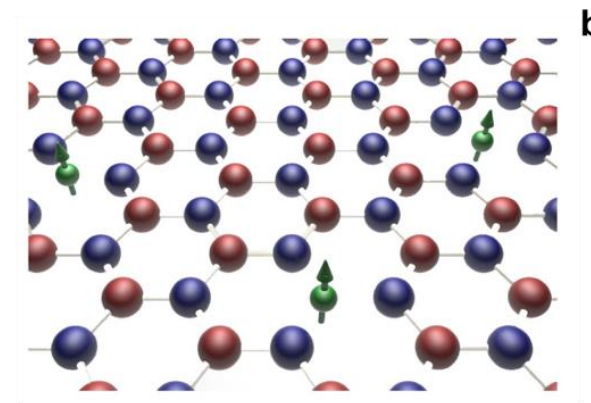

b

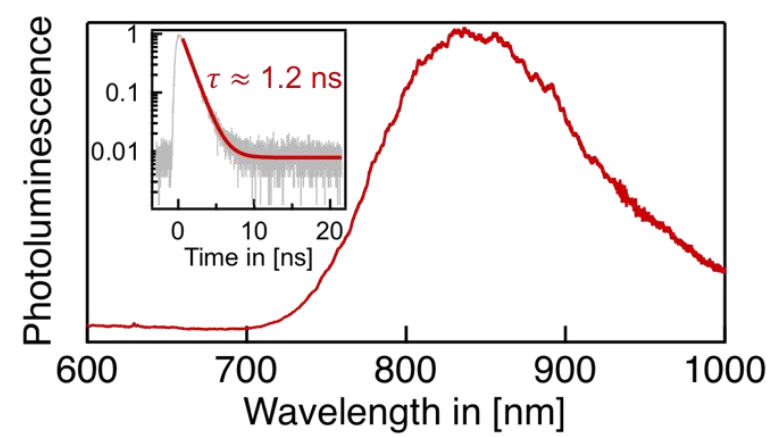

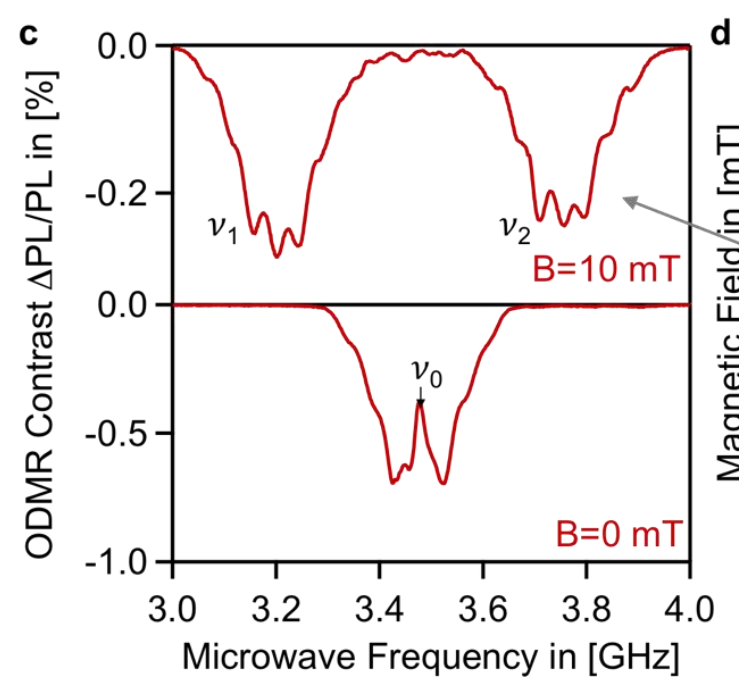
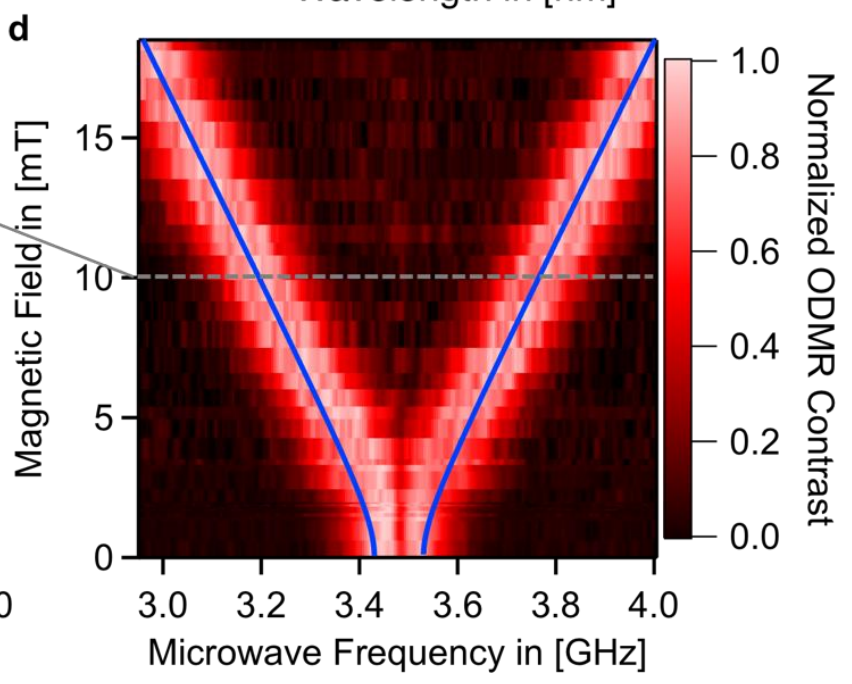

Figure 1. ODMR of an $h B N$ single crystal at room temperature, $T=300 \mathrm{~K}$. a) Schematic of an $h B N$ monolayer and its crystalline hexagonal structure with alternating boron (red) and nitrogen (blue) atoms. The green arrows indicate the spins of the negatively-charged boron-vacancy defects, $V_{B}^{-}$. b) Photoluminescence spectrum of the sample at room temperature displaying a pronounced emission at $850 \mathrm{~nm}$ with excited state lifetime of $1.2 \mathrm{~ns}$ (inset: PL transient). c) ODMR spectra measured with zero magnetic field (bottom) and with magnetic field $B=10 \mathrm{mT}$ (top); d) Dependence of ODMR frequencies $v_{1}$ and $v_{2}$ on the magnetic field (B/lc). Experimental data (red) and fit (blue line) obtained using Equation 2 with parameters $D / h=3.48 \mathrm{GHz}, E / h=50 \mathrm{MHz}$ and $g=2.000$. 
Most interestingly, we find that the PL from this hBN colour centre is spin-dependent. Figure 1c shows the ODMR spectrum recorded for an $\mathrm{hBN}$ single crystal at $\mathrm{T}=300 \mathrm{~K}$. In ODMR experiments, microwave-induced magnetic dipole transitions between spin-sublevels manifest as changes in $\mathrm{PL}$ intensity $(\triangle \mathrm{PL})$. The prerequisite for optical $(\mathrm{PL})$ detection of EPR is thus the existence of a dependence between the optical excitation-recombination cycle and the defect's spin orientation. Figure 1c shows the spectrum of the investigated sample as normalized change of $\mathrm{PL}$ intensity $(\triangle \mathrm{PL} / \mathrm{PL})-$ i.e. ODMR contrast - as a function of the applied microwave frequency $v$ for two static magnetic fields $B=0$ and $\mathrm{B}=10 \mathrm{mT}$. Even without external magnetic field, the ODMR spectrum shows two distinct resonances $v_{1}$ and $v_{2}$, located symmetrically around the frequency $v_{0}$. We tentatively assign them to the $\Delta m_{S}= \pm 1$ spin transitions between triplet energy sublevels with completely lifted three-fold degeneracy, due to a splitting induced by dipolar interaction between the unpaired electron spins, forming the triplet. This socalled zero field splitting is described by parameters $D$ and $E$ which can be derived from the spectrum as $D / h=v_{0}$ and $v_{1,2}=(D \pm E) / h$, with $h$ being Plank's constant to express the interaction energies in frequency units. In order to verify this assignment, we have studied the dependence of the $v_{1}$ and $v_{2}$ resonant microwave frequencies on the magnitude of the external static magnetic field.

The evolution of the ODMR spectrum with the field applied parallel to the hexagonal $\boldsymbol{C}$-axis $(\boldsymbol{B} \| \boldsymbol{C})$ of $\mathrm{hBN}$ is presented in Figure 1d. To explain the observed transitions and their variation with magnetic field, we use the standard spin Hamiltonian given by Equation 1 with $Z$ as the principle symmetry axis oriented perpendicular to the plane (collinear with the $c$-axis of the hBN crystal).

$$
H=D\left(S_{z}^{2}-S(S+1) / 3\right)+E\left(S_{x}^{2}-S_{y}^{2}\right)+g \mu_{B} \boldsymbol{B} \cdot \boldsymbol{S}
$$

where $D$ and $E$ are the ZFS parameters, $S=1$ is the total spin, $g$ is the Lande factor, $\mu_{B}$ is the Bohr magneton, $\boldsymbol{B}$ is the static magnetic field and $S_{x, y, z}$ are the spin-1 operators. According to Equation 1 and for $\boldsymbol{B}$ applied parallel to the $c$-axis the resonant microwave frequencies at which the transitions occur vary as

$$
v_{1,2}=v_{0} \pm \frac{1}{h} \sqrt{E^{2}+\left(g \mu_{B} B\right)^{2}}
$$

where $v_{0}=D / h$. The dependence of ODMR frequencies $v_{1}$ and $v_{2}$ on the magnetic field shown in Figure $1 \mathrm{~d}$ can be perfectly fitted by Equation 2 with $g=2.000, D / h=3.48 \mathrm{GHz}$ and a small off-axial component of the ZFS $E / h=50 \mathrm{MHz}$. This demonstrates a highly symmetrical, almost uniaxial defect structure.

So far, we have shown that the investigated hBN defect is a $S=1$ system, which can be optically addressed and read out using ODMR. However, from the ODMR measurements alone, it is difficult to conclude whether an excited, metastable or ground state of the defect forms the triplet state. The latter is essential to know in order to determine the correct spin-dependent recombination pathway. We note that earlier results ${ }^{25}$ proposed a defect in $\mathrm{hBN}$ with a singlet ground state to possess a spin-triplet metastable state. However, from our EPR results presented below, it becomes clear that we are dealing with another defect here. A second consideration is that zero-field ODMR measurements on their own are not sufficient to deduce the microscopic structure of the defect. To address these points, we applied high-field ODMR and EPR to previously studied exfoliated hBN flakes ${ }^{26}$ as well as to the hBN single crystal as studied by ODMR in Figure 1. We also note that the defects in hBN single crystals and in the hBN-exfoliated flakes we introduced in the same way (see Methods), and we only see the PL emission at $800 \mathrm{~nm}$, and ODMR signal after implantation/neutron irradiation and annealing. These defects (while may be present) were not optically active in pristine samples. 
Figure 2a shows EPR spectra taken at a fixed microwave frequency, $v=9.4 \mathrm{GHz}$, while scanning the static magnetic field aligned parallel to the $c$-axis of the crystal $(\boldsymbol{B} \| \boldsymbol{C})$. Due to the amplitude modulation of the $B$ field, EPR spectra usually look like first derivatives of the absorption signals. The spectra are recorded with (green trace) and without (black trace) optical excitation and consist of two groups of signals originating from two different types of paramagnetic species. The first group (centred at $B \approx 330 \mathrm{mT}$ ) is characterised by $g=2.003$ and consists of three EPR lines of nearly equal intensities corresponding to a paramagnetic centre with electron spin $S=1 / 2$ interacting with a nuclear spin $I=1$. The origin of such a splitting is hyperfine interaction. This group of lines remains the same with and without optical excitation and is not observed in ODMR under the same conditions (see Supplementary Figure S1). So, we conclude that spin transitions that cause these EPR signals are optically inactive. Although spin $S=1 / 2$ centres in hBN were already reported in the late 1970 s and assigned to a oneboron-centre $(\mathrm{OBC})$ or three-boron-centre $(\mathrm{TBC})$ defect $^{27}$, we believe that we are dealing with a $S=1 / 2$ centre which occupies a boron site in the lattice that interacts with one ${ }^{14} \mathrm{~N}(I=1)$. However, this interpretation is beyond the scope of this work.

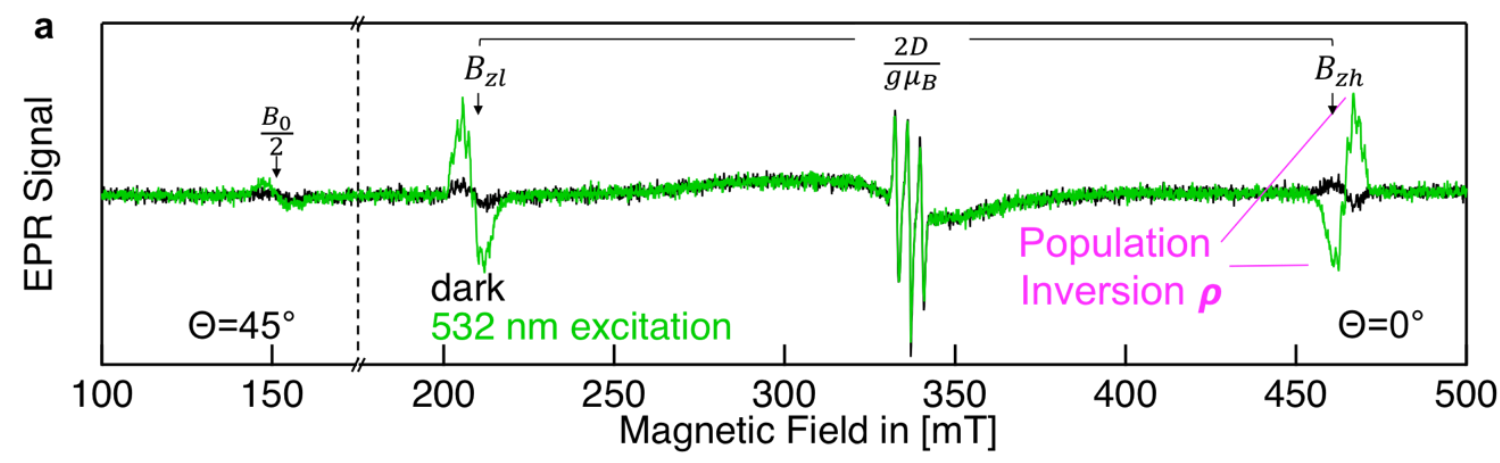

b

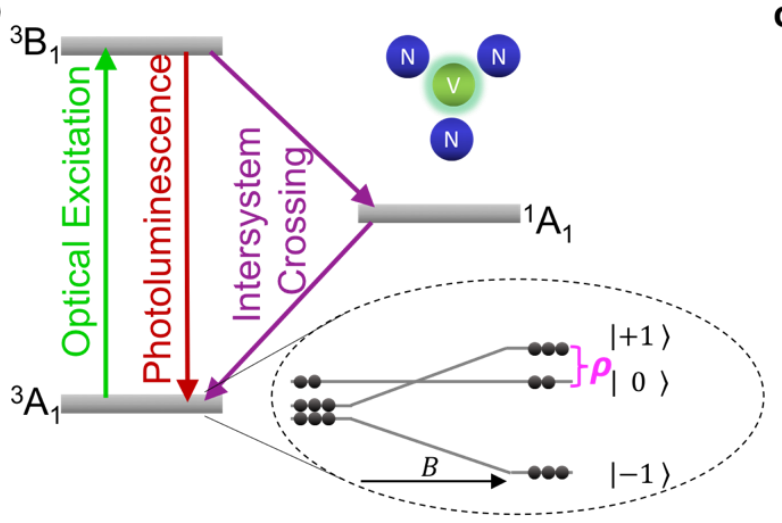

C

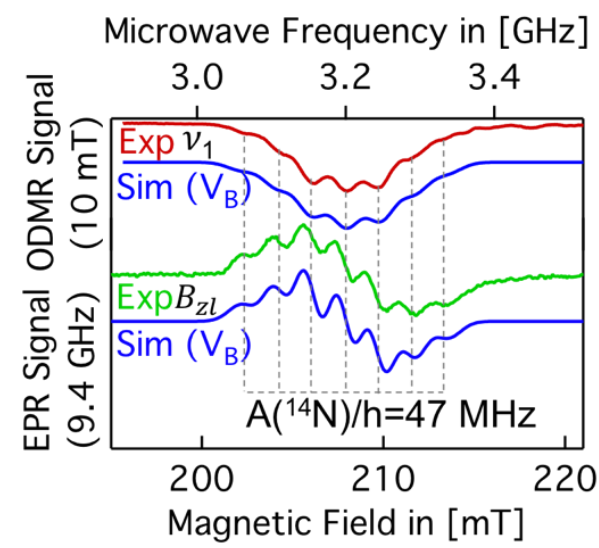

Figure 2. EPR studies of the $V_{B}^{-}$centre in the $h B N$ single crystal sample at $T=5 \mathrm{~K}$. a) EPR spectra measured with (green trace) and without (black trace) $532 \mathrm{~nm}$ optical excitation in the magnetic field orientation $\boldsymbol{B} / / \boldsymbol{c}$. Doublet lines associated with the $S=1$ centre with splitting of $\Delta B \approx 260 \mathrm{mT}$ (equivalent to $2 D / h \approx 2 \times 3.6 \mathrm{GHz}$ ) are labelled $B_{Z l}, B_{Z h}$. b) Simplified energy level diagram illustrating the optical spin polarization $\rho$ of the ground state with the optically pumped $m_{S}= \pm 1$ sublevels via intersystem crossing (ISC), c) Hyperfine splitting of EPR $B_{Z l}$ (green trace) and zero-field ODMR $v_{1}$ (red trace) lines. Vertical bars indicate seven transitions caused by hyperfine interaction with 3 equivalent nitrogen nuclei. The blue traces are the simulated EPR / ODMR spectra of the $\left(V_{B}^{-}\right)$defect with Eq.1, where the hyperfine term with a splitting constant $A / h=47 \mathrm{MHz}$ is considered.

On the other hand, the second group of EPR lines in Figure 2a (labelled $B_{Z l}, B_{Z h}$ with $I, h$ indices denoting low and high magnetic field transitions and the principal symmetry axis $Z$ the defect orientation $B$ I| $\boldsymbol{C}$ || Z) consists of two widely separated transitions, which are strongly responsive to optical excitation (532 nm laser). The splitting between the lines $B_{Z l}$ and $B_{Z h}(\Delta B \approx 260 \mathrm{mT})$ as well as the 
position of the $B_{0} / 2$ signals at half-field are the same as in ODMR experiments under the same conditions performed on the hBN flakes (see Supplementary Figure S1). The EPR signals can be satisfyingly described by the spin-Hamiltonian (Equation 1) with the following Zeeman and ZFS parameters: $S=1, g=2.000, D / h=3.6 \mathrm{GHz}$. The ZFS parameter $E$, which became apparent in ODMR measurements at $B=0$ (Figure 1c), could not be easily resolved in X-band EPR due to its small magnitude. It is however noticeable that the EPR measurements yield a slightly larger value for the ground-state ZFS parameter $D / h \approx 3.6 \mathrm{GHz}$ than what we determined from zero-field ODMR. This is expected for triplet spin centers ${ }^{23}$ and we attribute this difference to a pronounced temperature dependence of $D$ varying between $3.6 \mathrm{GHz}$ at $T=5 \mathrm{~K}$ (determined via EPR and ODMR) and $3.48 \mathrm{GHz}$ at $T=300 \mathrm{~K}(\mathrm{ODMR})$. Assuming a linear dependence, this would lead to a slope of approximately $0.4 \mathrm{MHz} / \mathrm{K}$, which can be reasonably expected for the temperature induced hBN lattice expansion.

Notably, the phases of the $B_{Z l}$ and $B_{Z h}$ signals shown in Fig. 2a become opposite (up-down and downup) upon optical excitation and we observe emission rather than absorption for the EPR transition $B_{Z h}$. This can be explained by an optically-induced population inversion $\rho$ taking place amongst the spintriplet sublevels of the ground state - with either the $m_{S}= \pm 1$ or $m_{S}=0$ sublevels being lower-lying at $B=0$. The order of the energy sublevels $m_{S}=0, \pm 1$ is determined by the sign of the ZFS parameter $D$, which has yet to be determined in our case.

In Figure $2 b$, we propose the tentative energy level scheme of the spin-defect consistent with our observations. Zeeman splitting of the $m_{S}= \pm 1$ levels results in the crossing of $m_{S}=+1$ and $m_{S}=0$ sublevels, while optical pumping induces population transfer (via excited and metastable states) from $m_{S}=0$ to $m_{S}= \pm 1$ and results in microwave emission at the $B_{Z h}$ field. We also want to emphasize that the EPR transitions between these triplet sublevels are also visible without optical excitation (black trace in Fig. 2a), suggesting that the triplet state we are looking at is in the ground state.

Although hBN can accommodate a large number of defects (either intrinsic or extrinsic) in its lattice sites, the combined EPR and ODMR data allow us to pinpoint the type of defect. The common defects include boron vacancies $\left(V_{B}\right)$, nitrogen vacancies $\left(V_{N}\right)$ and anti-site complexes (e.g. a nitrogen atom on a boron site next to a vacancy $\left(V_{N} N_{B}\right)$, or substitutional carbon-related defects like $C_{B}$ or even $\left.V_{N} C_{B}\right)$. The complex defects such as $V_{N} N_{B}$ and $V_{N} C_{B}$ were shown to have in-plane $C_{2 v}$ symmetry ${ }^{11,28}$, which is inconsistent with our observations of an almost axial defect with respect to the $c$-axis. On the other hand, there it was also shown that point defects $V_{B}, C_{B}$ and $V_{N}$ are characterized by the uniaxial $D_{3 h}$ group symmetry with a $\mathrm{C}_{3}$ rotation axis parallel to the $c$-axis. These defects are thus compatible with our findings and must be considered. Recent theoretical investigations of point defects in hBN have shown that the neutral $V_{N}^{0}$ and $C_{B}^{0}$ defects should be $S=1 / 2$, but they may become non-magnetic in their energetically preferred charge state $\left(V_{N}^{+}, V_{N}^{-}, C_{B}^{+}\right)$, while the negatively-charged boron vacancy $\left(V_{B}^{-}\right)$has been predicted to be stable with a $S=1$ ground state. ${ }^{29}$ In addition, the optically-induced spin polarisation of the $m_{S}= \pm 1$ levels by intersystem crossing (ISC) has already been theoretically proposed for the $V_{B}^{-}$triplet ground state ${ }^{11}$.

To discern between possible hBN lattice cites for the defect, we analysed the hyperfine structure of EPR and ODMR signals. Figure $2 c$ shows the $B_{Z l}$ EPR line together with the zero-field ODMR $v_{1}$ transition. The hyperfine splitting is known to be due to the interaction of the electron spin with the surrounding nuclear spins, sometimes called superhyperfine interaction - which in turn reflects the nature of the nearest atoms (in our case either three boron or three nitrogen atoms). It can be described by adding the term $\sum_{k} S A_{k} I_{k}$ to Equation 1 , where $A_{k}$ is the hyperfine interaction and $I_{k}$ is the nuclear spin. The number of observed hyperfine lines is seven (Fig. 3c). This is consistent with a spin $S=1$ point defect localized at the boron cite interacting with $n=3$ equivalent nitrogen atoms (nuclear spin $I=1,{ }^{14} \mathrm{~N}, 99.63 \%$ natural abundance) and it thus supports the cases of the $C_{B}$ and $V_{B}$ defects with $2 n I+1=7$ hyperfine transitions. Conversely, for $V_{N}$ there is hyperfine interaction of the electron spin with $\mathrm{n}=3$ equivalent boron atoms, each having two isotopes $\left(I=3 / 2,{ }^{11} \mathrm{~B}, 80.2 \%\right.$ natural abundance 
and $I=3,{ }^{10} \mathrm{~B}, 19.8 \%$ natural abundance), resulting in $2 n I+1=10$ plus $2 n I+1=19$ transitions. The $V_{N}$ simulation shown in Supplementary Figure $\mathrm{S} 2$ takes the natural isotope abundance into account and is dominated by the 10 hyperfine transitions for ${ }^{11} \mathrm{~B}$.

Consequently, ruling out $V_{N}$, the numerical simulations of the hyperfine structure agree well with a carbon atom or a vacancy localized at the boron cite (see Figure 2c and Supplementary Figure S2) yielding a hyperfine splitting constant $A / \mathrm{h}=47 \mathrm{MHz}$. Looking more closely, there are no obvious spectral features pointing at hyperfine interactions (HFI) with second neighbors (boron). These $\mathrm{n}=6$ equivalent boron atoms would have a much smaller coupling constant $A$ with $2 n I+1=20$ plus 38 transitions for ${ }^{10} \mathrm{~B}$ and ${ }^{11} \mathrm{~B}$, respectively. The spectral features would fall within the observed linewidth and would only result in slight broadening and miniscule modulations on top of the spectrum. Alternatively, the lack of obvious second neighbor HFI interaction could be a sign of a very localized wavefunction. Future simulations in combination with electron-nuclear double resonance experiments (ENDOR) could help to clarify the wavefunction extent.

Note, that while we can determine the defect symmetry $\left(D_{3 h}\right)$ and lattice site (boron), based on EPR data alone, we cannot fully discern whether the defect is intrinsic $\left(V_{B}\right)$ or extrinsic $\left(C_{B}\right)$. Consequently, we performed irradiation of pristine hBN material (cf. Methods) with various species: ion implantation with different ions (Lithium and Gallium) on hBN flakes, as well as neutron irradiation on exfoliated flakes and a hBN single crystal. The $800 \mathrm{~nm}$ PL band (Fig. 1b) can be observed for all of these samples (Supplementary Figure S4a). Furthermore, ODMR is detected (Supplementary Figure S1, S4b) for all but the Ga implanted flakes, due to damage from the Ga beam and very low PL intensity to yield an ODMR contrast. We can thus deduce that the investigated defect is indeed of intrinsic nature and is most likely the $V_{B}^{-}$.

To test the symmetry of the defect more closely, we analysed the angular dependencies of the EPR signals for rotations around the polar and azimuthal angles $\theta$ and $\phi$, respectively. Figure 3 shows the EPR signals measured for a polar rotation $(\theta)$ of the magnetic field from parallel $(\boldsymbol{B} \| \boldsymbol{C})$ to perpendicular $(\boldsymbol{B} \perp \boldsymbol{C})$ orientation and for azimuthal rotation $(\phi)$ in the $(0001)$ plane $(\boldsymbol{B} \perp \boldsymbol{C})$ of an hBN single crystal. The angular variation of the resonant magnetic field is described by numerical simulation of Eq. 1 employing the full set of the previously derived parameters $(S, g, D, E)$, as summarized in a Table in the Supporting Information. For both polar (Fig. 3a) and azimuthal rotation (Fig. 3b) we find exceptional good agreement of the overlaid simulated traces and the magnetic field positions of the experimentally observed transitions. We note that for the angular dependence measured in the 0001 plane $(B \perp \boldsymbol{C}$ in Fig. 3b), the splitting between the lines remains unchanged. These results point at the $c$-axis being the axis of symmetry of the almost uniaxial ODMR active triplet.
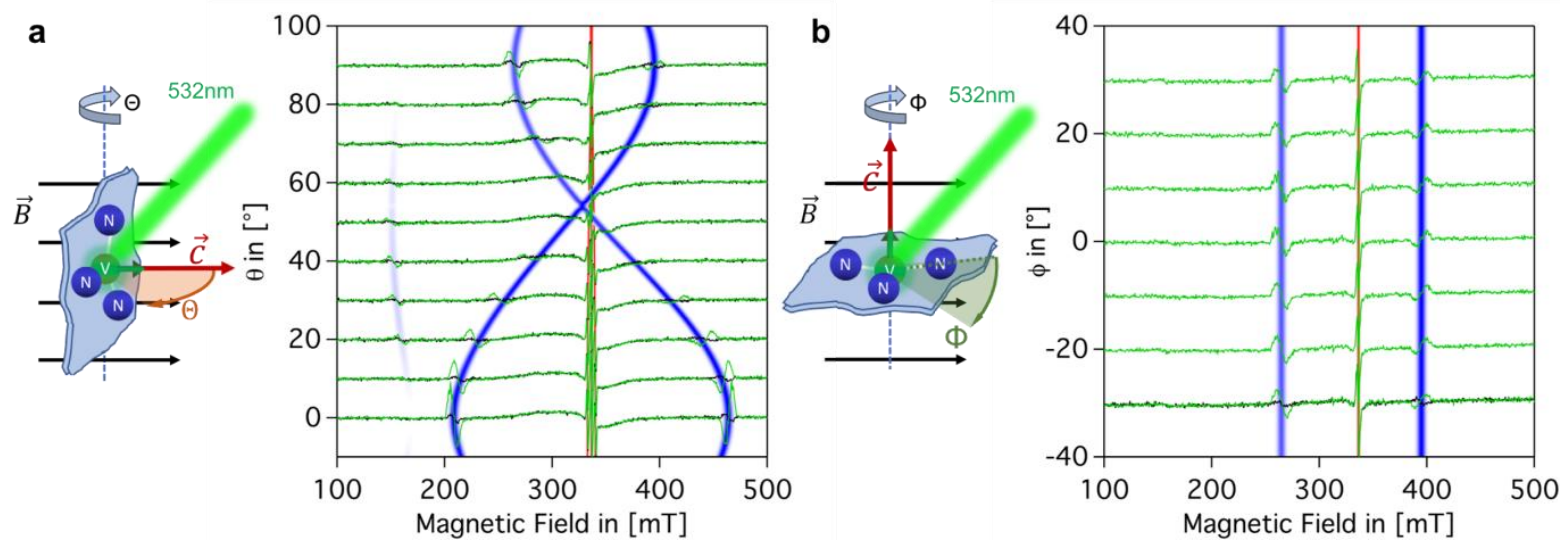

Figure 3. Angular dependence of the EPR spectra in an hBN single crystal. (a) Rotation of the magnetic field relative to the $\boldsymbol{C}$-axis from parallel $(\boldsymbol{B} / \mid \boldsymbol{C})$ to perpendicular $(\boldsymbol{B} \perp \boldsymbol{C})$ orientation and $(\boldsymbol{b})$ in the (0001) plane of $h B N(\boldsymbol{B} \perp \mathbf{C})$. Sketches explain the respective rotation schemes. Calculated angular dependencies are shown as blue traces. Angular variation of the $S=1 / 2$ centre (central EPR group, see text) is shown in red. The detailed analysis of this region in shown in Supplementary Figure S3. 
To conclude, we have demonstrated room-temperature optical initialisation and readout of ensembles of intrinsic $S=1$ defect centres in hBN. From rigorous EPR measurements performed on hBN single crystal, exfoliated and ion irradiated flakes, we propose the investigated defect to be the negativelycharged boron vacancy $V_{B}^{-}$. We further demonstrated spin polarization under optical excitation and optically-induced population inversion in the triplet ground state, which provides the basis for coherent spin-manipulation. While the research into spin defects always starts with ensembles, we are confident that in the future it will be possible to address individual $V_{B}^{-}$defects, particularly using super resolution techniques ${ }^{30}$. The main challenge lies in means to increase the quantum efficiency and engineer defects deterministically at various densities. One particularly promising approach is to use resonant excitation to select one or a few defects out of an ensemble at cryogenic temperatures ${ }^{31}$. This stimulates further research in hBN-based heterostructures for quantum sensing applications and drives interest into deterministically engineering single $V_{B}^{-}$centres. The work will also accelerate the research into the spinoptomechanics with $\mathrm{hBN}$, particularly given the established theoretical framework ${ }^{32}$ and advances in nanofabrication of resonators ${ }^{33}$. Further, with an increased control over isotopic purity, coherent manipulation of spin states may become feasible, yielding potentially high coherence times -as predicted theoretically ${ }^{34}$. In this context, the $V_{B}^{-}$defect identified here may be advantageous due to weaker coupling of the defect electron spin with the surrounding ${ }^{14} \mathrm{~N}$ nuclear bath compared to other possible defect configurations.

\section{Data availability}

All relevant data are available from the authors upon request.

\section{Acknowledgements}

V.D. acknowledges financial support from the DFG through the Würzburg-Dresden Cluster of Excellence on Complexity and Topology in Quantum Matter-ct.qmat (EXC 2147, project-id 39085490) and DY18/13-1. V.S. gratefully acknowledges the financial support of the Alexander von Humboldt $($ AvH) Foundation. The Australian Research council (via DP180100077, DP190101058 and DE180100810), the Asian Office of Aerospace Research and Development grant FA2386-17-1-4064, the Office of Naval Research Global under grant number N62909-18-1-2025 are gratefully acknowledged. I.A. is grateful for the Humboldt Foundation for their generous support. The authors are grateful for the neutron irradiation services at CTDN, Brazil.

\section{Methods}

\section{hBN samples}

Monocrystalline $\mathrm{hBN}$ and exfoliated flakes from $\mathrm{HQ}$ graphene were neutron irradiated in the Triga Mark I IPR-R1 nuclear reactor (CDTN, Brazil), with a thermal flux of $4 \cdot 10^{12} \mathrm{ncm}^{-2} \mathrm{~s}^{-1}$ for $16 \mathrm{~h}$ reaching an integrated dose of approximately $2.3 \cdot 10^{18} \mathrm{n} \mathrm{cm}^{-2}$. All the samples were irradiated in Cd capsules for which thermal neutrons are blocked, whilst the most energetic neutrons pass through the sample. The multilayered hBN flakes were irradiated with Lithium or Gallium ions, respectively to create the defects. The same luminescence features were observed after neutron irradiation, as well as lithium or gallium ion implantation (see Supplementary Figure S4a). Irradiation of the pristine hBN samples with different sources was conducted to test our hypothesis that the emitters are intrinsic in nature rather than due to inclusion of foreign atoms. Irrespective of the source, the same luminescence emission appears in the near infrared when the irradiated samples are excited with a green laser, similar to the one presented in Figure 1b. For more details about sample preparation by fast neutrons and thermal stability of produced defects see reference ${ }^{26}$.

\section{Zero-field ODMR}


The zero-field ODMR measurements were performed with a confocal microscope setup. For optical excitation, a $532 \mathrm{~nm}$ laser was coupled into a $50 \mu \mathrm{m}$ optical fibre and focussed onto the sample using a x10 objective (Olympus LMPLN10XIR), with the laser spot measuring approximately $10 \mu \mathrm{m}$ in diameter. The laser power at the surface of the sample was $10 \mathrm{~mW}$. The PL was then collected through the same objective and separated from the scattered laser light using a $650 \mathrm{~nm}$ short pass dichroic mirror and a $532 \mathrm{~nm}$ long pass filter. Behind the filter, it was coupled into a $600 \mu \mathrm{m}$ optical fibre and detected using a Si avalanche photodiode (Thorlabs APD120A). The sample was placed on a $0.5 \mathrm{~mm}$ wide copper-stripline to apply the microwaves generated by a signal generator (Stanford Research Systems SG384) and amplified by an amplifier (Mini Circuits ZVE-3W-83+). ODMR was detected by a Signal Recovery 7230 lock-in amplifier referenced by on-off modulation of the microwaves. A permanent magnet was mounted below the sample to generate the external magnetic field.

\section{EPR measurements}

EPR measurements were carried out with a modified Bruker spectrometer in the X-band regime $(9.4 \mathrm{GHz})$ with a microwave power of $20 \mu \mathrm{W}\left(\mathrm{B}_{1} \approx 0.5 \mu \mathrm{T}\right)$. The $\mathrm{hBN}$ sample was placed inside an optically-accessible microwave cavity on a rotatable quartz rod in order to change the angle with respect to the external magnetic field. Using an Oxford cryostat, the sample was cooled down to $5 \mathrm{~K}$. For optical excitation a $532 \mathrm{~nm}$ laser with $50 \mathrm{~mW}$ of power was directed through an optical window of the cavity.

\section{High-field ODMR}

X-Band $(9.4 \mathrm{GHz})$ ODMR measurements were performed in the same setup as for EPR using a second cavity window for optical readout. Transmitted light of the excitation wavelength is blocked by two filters (550 nm and $561 \mathrm{~nm}$ long pass) in order to detect the remaining broad PL of the emitting defects with a silicon photodiode (Hamamatsu S2281). The microwave source (Anritsu) is on-off modulated with $787 \mathrm{~Hz}$ and amplified to $2 \mathrm{~W}$. The PL signal is preamplified (Femto DLCPA-200) and recorded by a lock-in amplifier (Signal Recovery 7230). All EPR / ODMR spectral simulations were performed using EasySpin ${ }^{35}$. 


\section{Supplementary Information}

\section{Optical Initialisation and Readout of Intrinsic Spin Defects in a Hexagonal Boron Nitride at Room Temperature}

by A. Gottscholl et al.

\section{High-field ODMR on exfoliated hBN flakes}

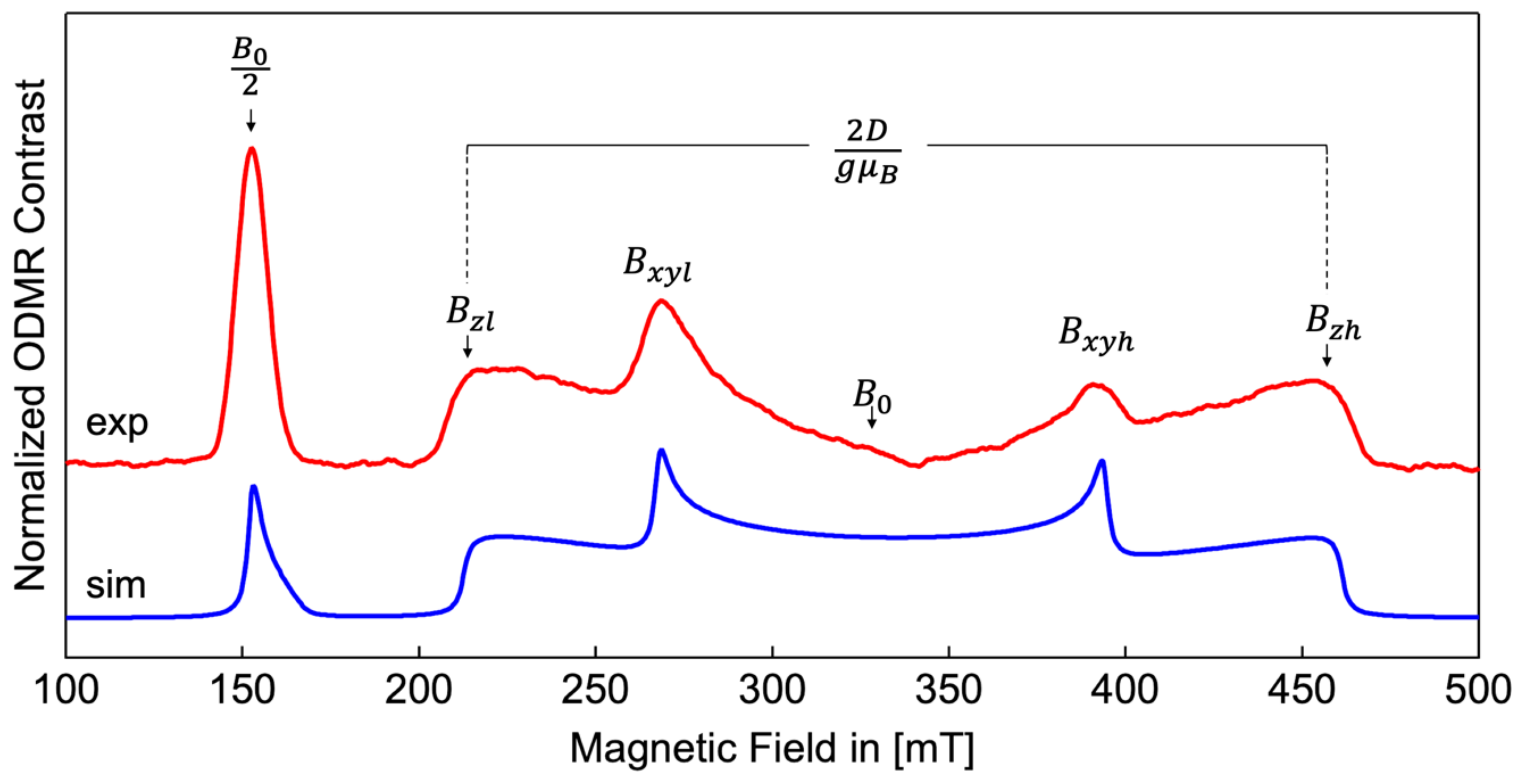

Supplementary Figure S1. High-field ODMR spectrum (red) obtained in randomly-oriented exfoliated $h B N$ flakes together with simulation (blue), displaying five peaks corresponding to changes in fluorescence intensity ( $\triangle P L / P L)$, as a function of the applied static magnetic field $B$. Four distinct resonance features corresponding to the selection rules $\Delta m_{s}= \pm 1$, which we label $B_{z l}, B_{z h}, B_{x y l}, B_{x y h}$ together with a "half-field" resonance at $B_{0} / 2$, corresponding to the double-quantum transition with $\Delta m_{s}= \pm 2$. The latter feature is often used to identify triplet states $(S=1)$ and to distinguish these from higher spin states. $D$ indicates the zero-field splitting parameter. $T=5 \mathrm{~K}$ and $\lambda_{\mathrm{exc}}=532 \mathrm{~nm}$.

The triplet ODMR spectrum shown in Supplementary Figure 1 fits perfectly to the EasySpin simulation of a randomly-oriented almost axial $S=1$, with the outer resonances corresponding to $\theta=0^{\circ}$ ( $B$ parallel to the $z$-axis), while the inner resonances correspond to $\theta=90^{\circ}$ ( $B$ orthogonal to the $z$-axis), respectively. The separation of the outer resonance lines is thus $2 \mathrm{D} / g \mu_{B}$. From the number of ODMR transitions and their magnetic field positions we conclude that we are probing randomly-oriented paramagnetic defects in the triplet state with almost axial symmetry, isotropic Landé factor $g \approx 2.000$ and ZFS value $\mathrm{D} / h=g \mu_{B}\left(B_{Z h}-B_{Z l}\right) /(2 h) \approx 3.6 \mathrm{GHz}$. The value of $E$ cannot directly be determined from this spectrum alone and is more accurately determined from the zero-field ODMR spectra in the main text. 
EPR experiment versus simulation of the hyperfine structure of negatively-charged boron vacancy and negatively-charged nitrogen vacancy in hBN single crystal
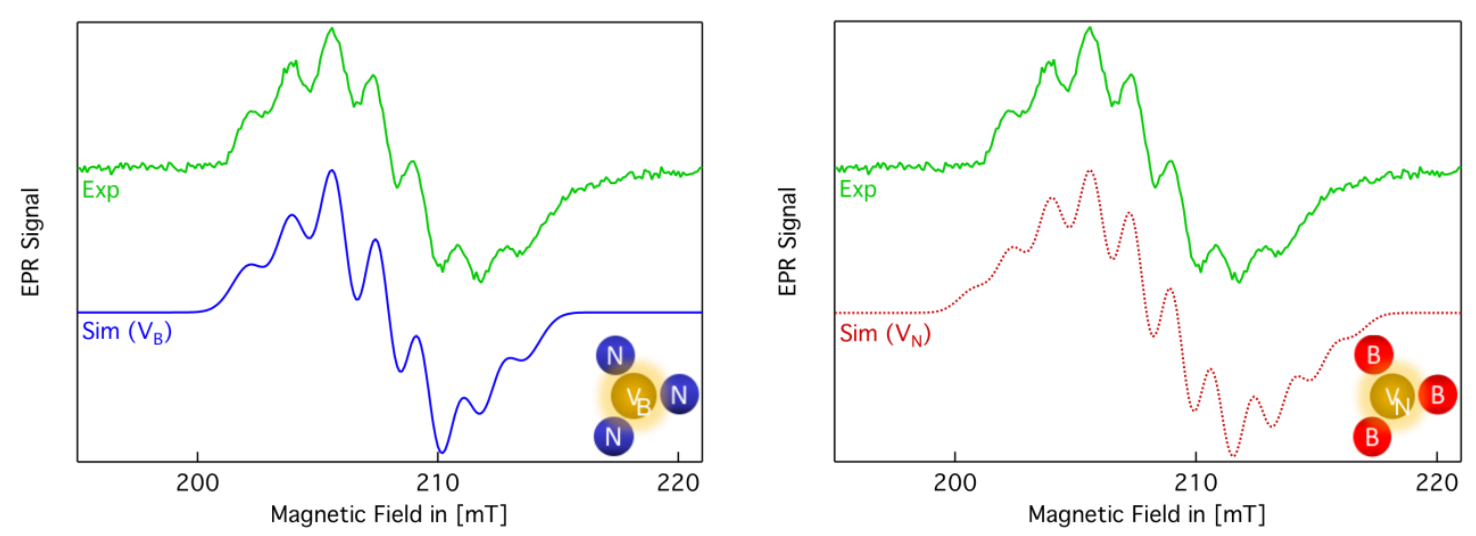

Supplementary Figure S2. Detailed depiction of Figure $2 c$ in the main text. The green curve shows the measured EPR signal for $532 \mathrm{~nm}$ excitation at $5 \mathrm{~K}$ for the $\boldsymbol{B} / / \boldsymbol{C}$ configuration. The blue line is an EasySpin simulation for a $S=1$ boron vacancy with a g-factor of 2.000, a peak-to-peak linewidth of $1.6 \mathrm{mT}$ and a zero-field splitting of $D / h=3.6 \mathrm{GHz}$ surrounded by three equivalent nitrogen nuclei with hyperfine coupling of $A / h=47 \mathrm{MHz}$. The seven transitions of the measurement fit the expected number of peaks $(2 \mathrm{nI}+1=7)$ for three equivalent nitrogen atoms with a nuclear spin of $I=1\left({ }^{14} \mathrm{~N}, I=1\right.$, $99.63 \%)$. To verify the compatibility of the EPR results with the nitrogen vacancy model, we show the EasySpin simulation for a nitrogen vacancy surrounded by three equivalent boron atoms (red line). For boron we consider the two isotopes with their natural abundance ${ }^{11} \mathrm{~B}(I=3 / 2,80.2 \%)$ and ${ }^{10} \mathrm{~B}(\mathrm{I}=3$, $19.8 \%)$ with ${ }^{11} B$ dominating the signal with $(2 n l+1=10)$ hyperfine transitions. The fit provides a slightly anisotropic hyperfine coupling, $A / h=[45.5 ; 44.0 ; 47.3] \mathrm{MHz}$ and ultimately does not reproduce the experimentally observed seven EPR transitions.

\section{Angular variation of the $S=1 / 2$ centre in $h B N$ single crystal shown in Figure 2}
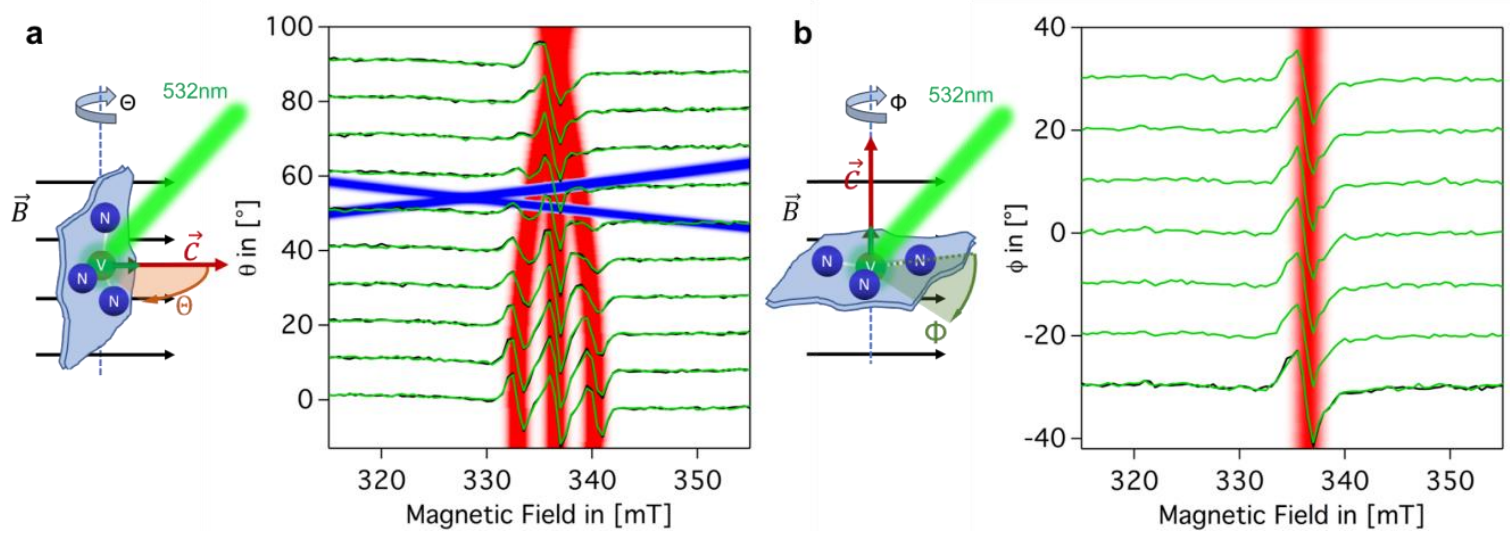

Supplementary Figure S3. Closer look at the central $S=1 / 2$ defect in the angular-dependent EPR measurements of Figure 3 in the main text. a) Case for the magnetic field parallel to the $h B N c$-axis $(\boldsymbol{B} / / \boldsymbol{C})$. b) Case for the magnetic field perpendicular to the $h B N c$-axis $(\boldsymbol{B} \perp \boldsymbol{c})$. The $c$-axis corresponds to the out-of-plane axis orthogonal to the $\mathrm{hBN}$ plane. $532 \mathrm{~nm}$ laser excitation is performed at $T=5 \mathrm{~K}$ (green spectra). However, the EPR intensities are independent of the excitation (black spectra). The red curves are EasySpin simulations for a $S=1 / 2$ defect with a $g$-factor of $g=2.003$ and an anisotropic hyperfine coupling $(A=[7.7 ; 28.5 ; 103.4] \mathrm{MHz})$ to one nitrogen atom $\left({ }^{14} \mathrm{~N}, I=1,99.63 \%\right)$. A deviation between simulation and measurement is observable for $\boldsymbol{B} \perp \boldsymbol{C}$ which leads to the conclusion that the assumption of an isotropic g-factor is not the best choice and thus a g-tensor has to be considered for a more detailed characterization of the defect. 


\section{$P L$ and ODMR spectra of various $h B N$ samples}

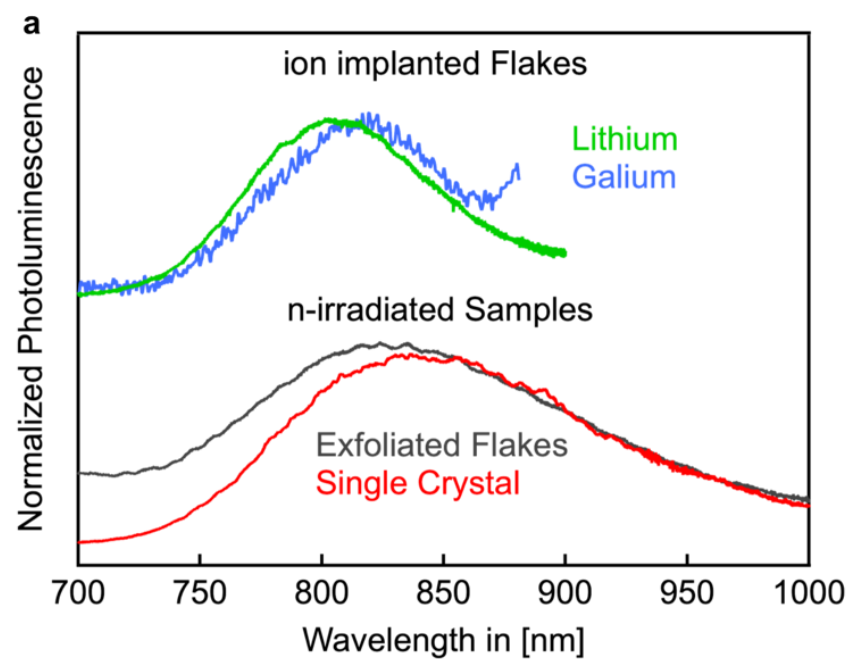

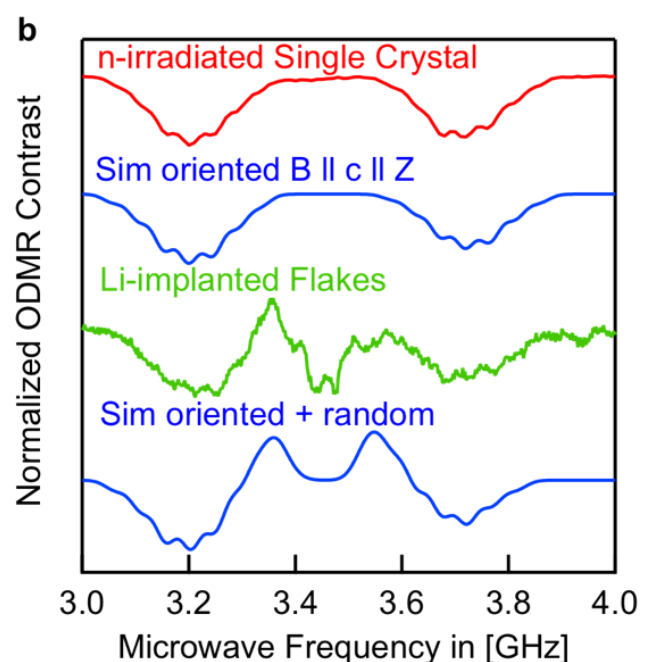

Supplementary Figure S4. Comparison of PL and ODMR spectra of various $h B N$ samples at room temperature and $\lambda_{e x c}=532 \mathrm{~nm}$.

a) PL spectrum of the neutron irradiated single crystal (same as Fig. $1 \mathrm{~b}$ of the main text) together with spectra of neutron irradiated exfoliated flakes (same as Fig. S1) and ion implanted flakes (both on glass substrate). All samples show a similar $P L$ at $\lambda_{\max } \approx 800 \mathrm{~nm}$. The glass substrate $P L$ at $>900 \mathrm{~nm}$ superimposes strongly with the $h B N P L$ band at $800 \mathrm{~nm}$, therefore only the central part of the spectra is shown. PL was measured with a confocal Raman microscope with $532 \mathrm{~nm}$ excitation (Horiba Labram HR800).

b) ODMR spectrum at $10 \mathrm{mT}$ of the neutron irradiated single crystal with Easyspin simulation (same as Fig. $1 c$ of the main text) together with the spectrum of Lithium ion implanted flakes on glass substrate. The Li-implanted flakes show the same $v_{1}$ and $v_{2}$ resonances as the single crystal. Additionally, an asymmetrical ODMR signal at $v_{0} \approx 3.5 \mathrm{GHz}$ is visible. We tentatively attribute this central peak in the Li-implanted flakes to the same spin defects found in single crystals, but randomly oriented at the edges and surfaces. Strong angular dependence of EPR transitions known for single crystals (see Fig. 3a) may result in a spectral collapse of $v_{1}$ and $v_{2}$ resonances. To backup this spectrum with simulations, we calculated the superposition of the simulation of the oriented sample with an additional spectrum using the same EPR parameters - but with randomly oriented spin-defects. While the simulation clearly lacks the perfect reproduction of the signal contributions' signs and relative intensities of the spectral features, the additional spectral feature in the center can be identified. 
The parameters of all $V_{B}^{-}$EPR / ODMR simulations are summarized in the following together with the method which is best suited to derive that parameter accurately and the corresponding figures.

\begin{tabular}{|l|l|l|l|}
\hline Parameter & Value & most accurate from & Corresponding Figures \\
\hline$S$ & 1 & EPR, ODMR at B $>0$ & $1 \mathrm{~d}, 2 \mathrm{a}, 3, \mathrm{S1}, \mathrm{S} 4 \mathrm{~b}$ \\
\hline$g$ & 2.000 & ZF ODMR & $1 \mathrm{~d}$ \\
\hline$D / h$ & $3.48 \mathrm{GHz}$ & ZF ODMR at 300 K & 1 \\
\hline$D / h$ & $3.6 \mathrm{GHz}$ & HF EPR, ODMR at $5 \mathrm{~K}$ & $2 \mathrm{a}, 3, \mathrm{~S} 1$ \\
\hline$E / h$ & $50 \mathrm{MHz}$ & ZF ODMR & $1 \mathrm{~d}$ \\
\hline$A / h \quad$ & $47 \mathrm{MHz}$ & ZF ODMR, HF EPR & $2 \mathrm{c}, \mathrm{S} 2 \mathrm{a}$ \\
\hline $\begin{array}{l}\text { Spin center } \\
\text { orientation Z }\end{array}$ & $\begin{array}{l}\text { Z II c (defects } \\
\text { are in plane) }\end{array}$ & $\begin{array}{l}\text { angular dependent } \\
\text { EPR of single crystal }\end{array}$ & 3 \\
\hline $\begin{array}{l}\text { Spin center } \\
\text { orientation Z }\end{array}$ & $\begin{array}{l}\text { randomly } \\
\text { oriented }\end{array}$ & $\begin{array}{l}\text { HF, ZF ODMR of } \\
\text { exfoliated flakes }\end{array}$ & $\mathrm{S1}, \mathrm{S} 4 \mathrm{~b}$ \\
\hline
\end{tabular}




\section{References}

1 Qian, X., Liu, J., Fu, L. \& Li, J. Quantum spin Hall effect in two-dimensional transition metal dichalcogenides. Science 346, 1344-1347, (2014).

2 Urbaszek, B. \& Srivastava, A. Materials in flatland twist and shine. nature 567, 39, (2019).

3 Caldwell, J. D. et al. Photonics with hexagonal boron nitride. Nature Reviews Materials 4, 552-567, (2019).

4 Toth, M. \& Aharonovich, I. Single Photon Sources in Atomically Thin Materials. Annual Review of Physical Chemistry 70, null, (2019).

5 Tran, T. T., Bray, K., Ford, M. J., Toth, M. \& Aharonovich, I. Quantum emission from hexagonal boron nitride monolayers. Nature Nanotech. 11, 37-41, (2016).

6 Tran, T. T. et al. Robust Multicolor Single Photon Emission from Point Defects in Hexagonal Boron Nitride. ACS Nano 10, 7331-7338, (2016).

7 Jungwirth, N. R. et al. Temperature Dependence of Wavelength Selectable ZeroPhonon Emission from Single Defects in Hexagonal Boron Nitride. Nano Lett. 16, 6052-6057, (2016).

8 Shotan, Z. et al. Photoinduced Modification of Single-Photon Emitters in Hexagonal Boron Nitride. ACS Photonics 3, 2490-2496, (2016).

9 Kianinia, M. et al. Robust Solid-State Quantum System Operating at $800 \mathrm{~K}$. ACS Photonics 4, 768-773, (2017).

10 Tawfik, S. A. et al. First-principles investigation of quantum emission from $\mathrm{hBN}$ defects. Nanoscale 9, 13575-13582, (2017).

11 Abdi, M., Chou, J.-P., Gali, A. \& Plenio, M. B. Color Centers in Hexagonal Boron Nitride Monolayers: A Group Theory and Ab Initio Analysis. ACS Photonics, (2018).

12 Vogl, T., Campbell, G., Buchler, B. C., Lu, Y. \& Lam, P. K. Fabrication and Deterministic Transfer of High-Quality Quantum Emitters in Hexagonal Boron Nitride. ACS Photonics 5, 2305-2312, (2018).

13 Proscia, N. V. et al. Near-deterministic activation of room temperature quantum emitters in hexagonal boron nitride. Optica 5, 1128, (2018).

$14 \mathrm{Li}, \mathrm{X}$. et al. Nonmagnetic Quantum Emitters in Boron Nitride with Ultranarrow and Sideband-Free Emission Spectra. ACS Nano 11, 6652-6660, (2017).

15 Exarhos, A. L., Hopper, D. A., Grote, R. R., Alkauskas, A. \& Bassett, L. C. Optical Signatures of Quantum Emitters in Suspended Hexagonal Boron Nitride. ACS Nano 11, 3328-3336, (2017).

16 Atatüre, M., Englund, D., Vamivakas, N., Lee, S.-Y. \& Wrachtrup, J. Material platforms for spin-based photonic quantum technologies. Nature Reviews Materials 3, 38-51, (2018).

17 Gao, W. B., Imamoglu, A., Bernien, H. \& Hanson, R. Coherent manipulation, measurement and entanglement of individual solid-state spins using optical fields. Nat. Photonics 9, 363-373, (2015).

18 Yılmaz, S. T., Fallahi, P. \& Imamoğlu, A. Quantum-Dot-Spin Single-Photon Interface. Phys. Rev. Lett. 105, 033601, (2010).

19 Kalb, N. et al. Entanglement distillation between solid-state quantum network nodes. Science 356, 928-932, (2017).

20 Mamin, H. J. et al. Nanoscale Nuclear Magnetic Resonance with a Nitrogen-Vacancy Spin Sensor. Science 339, 557-560, (2013).

21 Aslam, N. et al. Nanoscale nuclear magnetic resonance with chemical resolution. Science 357, 67-71, (2017).

22 Lovchinsky, I. et al. Nuclear magnetic resonance detection and spectroscopy of single proteins using quantum logic. Science 351, 836-841, (2016).

23 Doherty, M. W. et al. The nitrogen-vacancy colour centre in diamond. Physics Reports 528, 1-45, (2013).

24 Geim, A. K. \& Grigorieva, I. V. Van der Waals heterostructures. Nature 499, 419-425, (2013). 
25 Exarhos, A. L., Hopper, D. A., Patel, R. N., Doherty, M. W. \& Bassett, L. C. Magneticfield-dependent quantum emission in hexagonal boron nitride at room temperature. Nat. Commun. 10, 222, (2019).

26 Toledo, J. R. et al. Electron paramagnetic resonance signature of point defects in neutron-irradiated hexagonal boron nitride. Phys. Rev. B 98, 155203, (2018).

27 Katzir, A., Suss, J. T., Zunger, A. \& Halperin, A. Point defects in hexagonal boron nitride. I. EPR, thermoluminescence, and thermally-stimulated-current measurements. Phys. Rev. B 11, 2370-2377, (1975).

28 Huang, B., Xiang, H., Yu, J. \& Wei, S.-H. Effective Control of the Charge and Magnetic States of Transition-Metal Atoms on Single-Layer Boron Nitride. Phys. Rev. Lett. 108, 206802, (2012).

29 Weston, L., wickramaratne, D., Mackoit, M., Alkauskas, A. \& van de Walle, C. G. Native point defects and impurities in hexagonal boron nitride. Phys. Rev. B 97, 214104, (2018).

30 Feng, J. et al. Imaging of optically active defects with nanometer resolution. Nano Lett 18, 1739, (2018).

31 Dietrich, A. et al. Observation of Fourier transform limited lines in hexagonal boron nitride. Phys. Rev. B 98, 081414, (2018).

32 Abdi, M., Hwang, M.-J., Aghtar, M. \& Plenio, M. B. Spin-Mechanical Scheme with Color Centers in Hexagonal Boron Nitride Membranes. Phys. Rev. Lett. 119, 233602, (2017).

33 Shandilya, P. K. et al. Hexagonal Boron Nitride Cavity Optomechanics. Nano Lett. 19, 1343-1350, (2019).

34 Ye, M., Seo, H. \& Galli, G. Spin coherence in two-dimensional materials. npj Computational Materials 5, 44, (2019).

35 Stoll, S. \& Schweiger, A. EasySpin, a comprehensive software package for spectral simulation and analysis in EPR. Journal of Magnetic Resonance 178, 42-55, (2006). 